\title{
L'enfant mort-né, du futur au plus-que-parfait
}

Child Born Without Life, of Future In the Past Perfect

Jean-Pierre Gasnier

\section{(2) OpenEdition}

Journals

Édition électronique

URL : https://journals.openedition.org/tc/6929

DOI : $10.4000 /$ tc. 6929

ISBN : 1952-420X

ISSN : 1952-420X

Éditeur

Éditions de l'EHESS

Édition imprimée

Date de publication : 19 juin 2013

Pagination : $30-43$

ISBN : 978-2-7351-1637-9

ISSN : 0248-6016

\section{Référence électronique}

Jean-Pierre Gasnier, «L'enfant mort-né, du futur au plus-que-parfait », Techniques \& Culture [En ligne],

60 | 2013, mis en ligne le 19 juin 2016, consulté le 29 septembre 2022. URL : http://

journals.openedition.org/tc/6929; DOI : https://doi.org/10.4000/tc.6929 


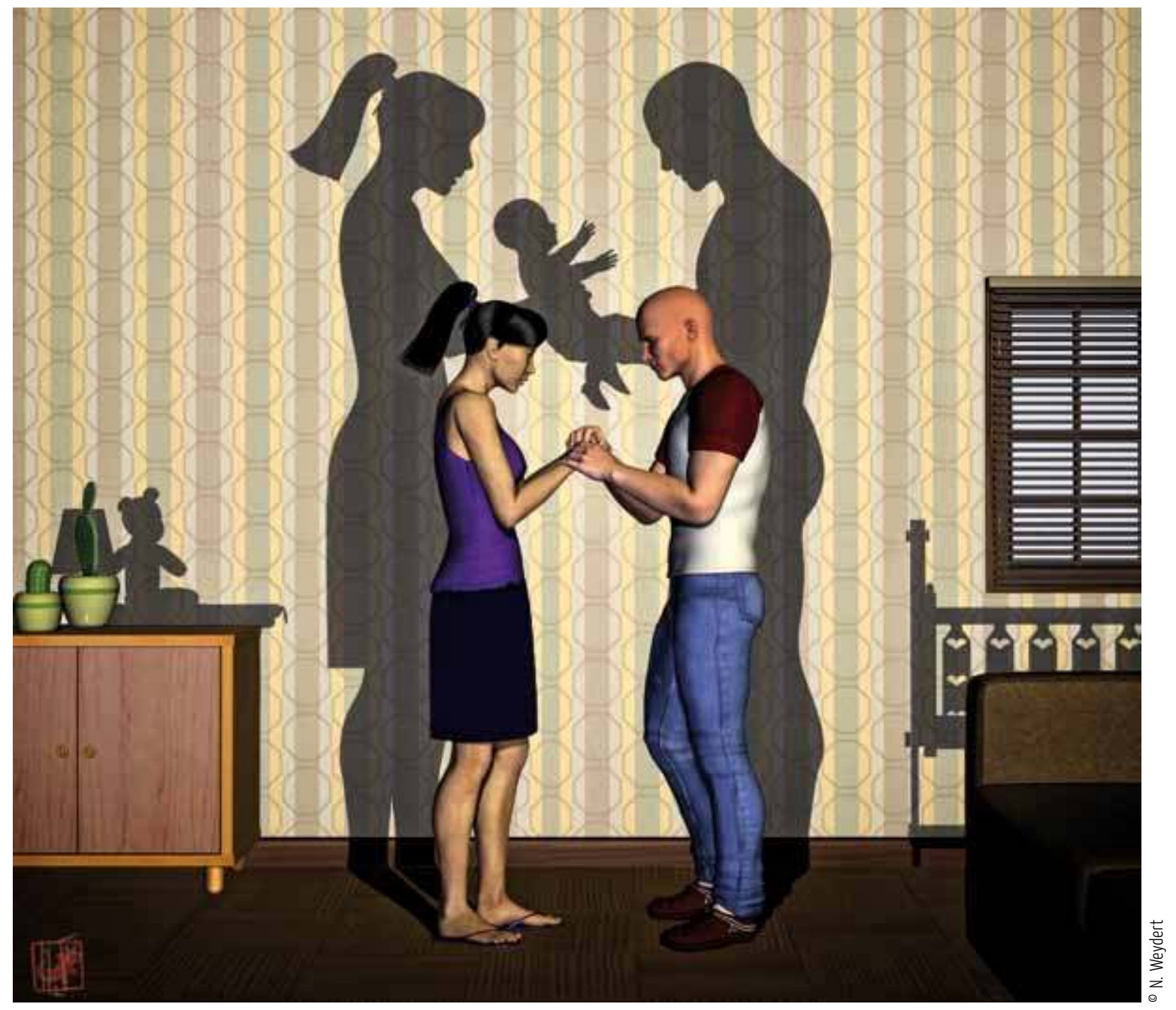




\section{L'ENFANT MORT-NÉ, DU FUTUR AU PLUS-QUE-PARFAIT}

L'enfant mort-né nous place d'emblée devant le fait qu'au cœur même de la procréation ily a la mort, devant le fait que donner la vie c'est donner la mort, puisque nous y sommes tous destinés. Seulement, avec l'enfant mort-né, un collapsus temporel s'opère puisque la mort est déjà là avant l'accouchement, ou survient au moment même de celui-ci.

Ceci n'est pas sans poser des questions éthiques, théoriques et pratiques aux juristes, lesquelles touchent à la notion même d'être humain et de personne. Ces questions, fréquemment discutées, sont rendues encore plus délicates du fait des progrès de la science, et plus particulièrement de la biologie qui permet de définir les divers stades de développement du zygote au fœetus. Jusqu'à présent, le droit parlait « d'enfant conçu » ou « d'enfant à naître ». Nous verrons plus avant que ces notions d'embryon et de foetus ne sont pas aussi étrangères à l'enfant mort-né qu'il n'y paraît.

Le Code civil, en introduisant dans son article 16 la notion « d'être humain » et de respect dû aux restes du corps humain, vient encore ajouter une notion supplémentaire: un embryon, un fœtus, enfants mort-nés sont-ils des «êtres humains », notion qui n'est pas définie par le Code civil, pas plus que par la Convention Européenne des Droits de l'Homme qui y fait pourtant abondamment référence. Une des questions posée aux juristes est en effet la suivante : à quel stade de développement peut-on parler d'être humain?

Ces questions donnent lieu à des discussions enflammées, à des prises de position doctrinales plus ou moins tranchées et militantes issues d'un débat agité qui concerne notamment le droit à l'avortement par opposition au droit à la vie, les techniques néonatales, etc. : un être humain ne peut-il être qu'un individu né et vivant, ou ayant 
été vivant un certain temps, ou peut-on attribuer cette qualification à un ensemble de cellules plus ou moins développé? Quelles sont les conséquences juridiques d'une telle qualification?

Une autre série de question tourne autour de la notion de personne telle qu'elle est traditionnellement entendue par le droit et va nous occuper ici: quand acquiert-on la personnalité juridique? Celle-ci est-elle liée à la naissance ou peut-elle être acquise intrautero, voire in vitro? La qualité de personne peut-elle faire l'objet d'un droit subjectif, d'un « droit à », ce qui revient alors à reconnaître à un embryon un droit à la vie, position qui n'est pas sans conséquence. Le Comité Consultatif National d'Éthique (CCNE), en proposant la notion de " personne potentielle ${ }^{1} »$ ne vient pas simplifier le débat. Le sort de l'enfant mort-né est donc directement conditionné par les réponses qui peuvent être données à ces questions, plus ou moins influencées par des prises de positions religieuses, philosophiques, morales, épistémologiques ou anthropologiques.

En outre, ces réponses ne seront pas sans influence, au-delà du droit civil; des points de vue du droit pénal: « peut-il y avoir homicide sur un fotus? », du droit social: « les parents peuvent-ils bénéficier de congés de maternité en cas d'enfant mort-né ou en cas d'interruption volontaire de grossesse? », du droit administratif: « que fait-on du corps d'un enfant décédé? Traite-t-on différemment le corps d'un enfant issu d'une IVG et celui d'un enfant mort-né? », du droit médical, notamment au regard de la bioéthique: « peut-on utiliser les tissus d'embryons morts, dans quelle mesure? », etc.

Pour entrer maintenant, directement, au cœur du sujet, il convient de s'interroger tout d'abord sur la notion même d'enfant mort-né, ce qui nous conduit à tenter de définir son statut juridique. Notre démonstration devra établir que l'enfant mort-né ou plus exactement né sans vie, selon la terminologie aujourd'hui retenue, est un reste. Il conviendra ensuite d'examiner le traitement réservé au corps de ces enfants, c'est-à-dire le traitement de ce reste. Mais, au-delà des textes et des classifications juridiques, il nous faut encore nous interroger sur le besoin qui se fait sentir, de façon persistante semble-t-il, d'en passer soit par des traces de l'enfant, soit par son corps, reste matériel et concret, pour penser et admettre la mort.

\section{L’enfant né sans vie: un reste}

Pour comprendre tout le débat juridique et l'enjeu de celui-ci, il faut savoir que le droit civil n’accorde la personnalité juridique qu'aux enfants nés vivants et viables ${ }^{2}$.

Un enfant est considéré comme vivant si, à la naissance, il a respiré, et viable, s'il possède les organes essentiels lui permettant d'avoir une existence autonome, c'est-à-dire qu'il peut vivre, même un bref instant, sans assistance technique externe.

Concernant le critère de viabilité, l'Organisation mondiale de la santé (OMS), dès 1977, a estimé qu'un enfant devait être considéré comme viable s'il est né après 22 semaines d'aménorrhée ou s'il a atteint le poids de 500 grammes, ces critères étant alternatifs et non cumulatifs.

Dès lors qu'un enfant est né vivant et viable, il possède la personnalité juridique, il dispose à ce titre d'une identité, par conséquent, d'un état civil et des droits, et cela, quand bien même il décéderait très rapidement après l'accouchement. Autrement dit, être né 
vivant et viable, même brièvement, confère à un enfant le statut juridique de personne. Il résulte de cet état de fait une obligation de déclarer l'enfant, dans les trois jours de la naissance, auprès de l'officier d'état civil. Cette déclaration doit être faite en principe par les parents (souvent le père) ou par le médecin ou la sage-femme ou toute personne ayant eu connaissance de la naissance (article 56 du Code civil). Si la déclaration n'a pas eu lieu dans les trois jours, l'officier d'état civil ne peut plus porter l'enfant sur les registres de l'état civil et il faut alors obtenir un jugement déclaratif de naissance. Cette exigence d'un état civil est essentielle puisque ce sont sur les registres de l'état civil que figurent les mentions relatives à ce que nous appelons « l'état de la personne » (identité, filiation, nom de famille, régime matrimonial, décès). Ces actes, qui font l'objet d'une publicité, attestent de l'existence juridique d'une personne et permettent de faire la preuve de cette existence tout en informant les tiers sur l'état de cette personne: est-elle majeure, célibataire, mariée, pacsée, etc.

Comme le souligne un auteur, l'état civil est un régime de publicité qui permet «au droit de différencier les individus et de fixer les frontières de leur existence » (Murat 1995), d'où cette exigence de déclaration et d'une déclaration rapide, pour ne pas laisser un enfant sans existence légale, ni sans droits.

Lorsqu'un enfant naît vivant, deux cas de figure peuvent donc se présenter.

Premier cas: l'enfant naît vivant et il est encore vivant au moment de la déclaration de naissance auprès de l'état civil. S'il décède ultérieurement, ce décès sera traité comme pour toute autre personne selon le droit funéraire en vigueur au moment du décès et un acte de décès sera établi.

Deuxième cas: l'enfant naît vivant mais décède avant la déclaration de naissance, c'est alors la notion de viabilité qui doit être prise en compte pour savoir si l'enfant dispose d'une personnalité juridique et s'il doit être inscrit comme enfant sur les registres d'état civil. S'il est déclaré viable, un acte de naissance et un acte de décès seront alors établis. Dans le cas contraire, l'enfant ne pourra pas être inscrit sur les registres d'état civil comme un enfant vivant.

Que se passe-t-il alors si l'enfant né vivant n'est pas viable ou lorsqu'un enfant naît déjà mort, c'est-à-dire qu'une femme donne naissance à un « enfant sans vie », selon la terminologie désormais adoptée? Tout le débat tourne précisément autour de l'inscription sur les registres de l'état civil et de la façon dont on doit y inscrire ces enfants, ce qui conditionne la reconnaissance de la personnalité juridique, ou son absence. Le débat n'est pas nouveau. En effet, la « déclaration du Roy ${ }^{3}$ » faite par Louis XV en 1736, traitait déjà de cette question. Après la Révolution, il faudra attendre un décret du 4 juillet $1806^{4}$ pour que la question soit abordée. Ce décret disposait que: « Lorsque le cadavre d'un enfant dont la naissance n'a pas été enregistrée sera présenté à l'officier de l'état civil, cet officier n'exprimera pas qu'un tel enfant est décédé, mais seulement qui lui a été présenté sans vie. Il recevra de plus la déclaration des témoins touchant les noms, prénoms, qualités et demeure des père et mère de l'enfant, et la désignation des an, jour et heure auquel l'enfant est sorti du sein de sa mère » (art. 1). Larticle 2 du même décret prévoyait également: «Cet acte sera inscrit à sa date sur les registres de décès, sans qu'il en résulte aucun préjugé sur la question de savoir si l'enfant a eu vie ou non ».

Autrement dit, dès 1806, il était fait mention de l'existence d'un « enfant sans vie » sur les registres d'état civil, sans qu'il soit fait mention du fait que l'enfant ait été vivant et viable et donc, sans préjuger du fait qu'il ait pu disposer, ne serait-ce qu'un instant, de la personnalité juridique avec les conséquences, notamment successorales, que cela 
peut entraîner. Toutefois, les juges ont rapidement été saisis de la question de savoir si tous les accouchements d'enfants ou de foetus mort-nés devaient être déclarés à l'état civil, ou si n'étaient concernés que ceux ayant donné lieu à l'expulsion d'un fotus après un certain stade de développement.

La Cour de cassation dans un arrêt du 7 août 1874 a ainsi décidé que seuls les enfants mort-nés viables (expression quelque peu paradoxale) devaient être présentés à l'officier d'état civil:

«Attendu que l'article 345 \& 3 du code pénal qui punit de six jours à deux mois de prison la suppression de l'enfant lors même qu'il est bien établi qu'il n'a pas vécu, doit être combiné avec l'article 312 du code civil, aux termes duquel l'enfant n'est réputé viable qu'après un minimum de cent quatre-vingts jours, ou six mois de gestation; que l'être qui vient au monde avant ce terme, privé non seulement de la vie, mais des conditions organiques indispensables à l'existence, ne constitue qu'un produit innommé et non un "enfant", dans le sens que le législateur a attaché à cette expression; que ce n'est point en vue d'un pareil être qui, suivant que sa venue au jour se rapproche davantage de l'époque de la conception, peut ne pas même présenter les signes distinctifs de la forme humaine que le décret du 3 juillet 1806 a prescrit la présentation du cadavre de tout enfant mort-né à l'officier d'état civil; qu’une telle présentation, sans utilité pour l'intérêt social, pourrait dans certains cas, blesser la pudeur publique. »

On ne manquera pas de noter la formulation de « produit innommé » pour désigner l'enfant mort-né, non viable au sens des critères retenus, qui fait basculer le mort-né du côté de quelque chose d'inclassifiable au regard des catégories juridiques, ni chose - (les juges auraient certainement employé le terme, aussi choquant puisse-t-il paraître), ni personne, - selon la traditionnelle distinction du Code civil entre personnes, choses et obligations, héritée du droit romain -, mais produit, - sans nom -, c'est-à-dire, tout à la fois, sans nom de famille, mais également sans nom pour le désigner en tant que réalité.

Autrement dit, sous l'empire du décret de 1806, deux cas de figure pouvaient se présenter: soit on avait affaire à un enfant né avant 180 jours de gestation. Présumé non viable celui-ci ne pouvait faire l'objet d'aucune déclaration, même au titre d'un acte d'enfant sans vie. Cette mesure était justifiée par le fait que, pour reconnaître une personnalité juridique, il fallait un enfant vivant et viable, et que dès lors il n'y avait pas lieu de garder trace à l'état civil de l'existence d'un être qui, en tout état de cause, n'aurait pu avoir de personnalité juridique; soit, parmi les enfants décédés avant la déclaration de naissance, on avait affaire à un enfant présumé viable car né après une grossesse ayant duré au moins 180 jours ou 6 mois et alors un acte d'enfant sans vie pouvait être établi.

Toutefois, cette hypothèse couvrait plusieurs situations très différentes: l'enfant était né vivant et viable, mais était décédé avant la déclaration de sa naissance. Il avait donc eu, momentanément, une personnalité juridique qui n'était pas reconnue. L'enfant était né vivant mais non viable en raison par exemple d'une malformation (et non au regard du délai de gestation), ce qu'on appelle un « faux mort-né ». L'enfant était né viable au regard du critère du délai de gestation, mais était décédé, soit au moment de l'accouchement, soit in utero.

Cette situation d'inexistence légale a perduré jusqu'à la fin du xxe siècle. Elle était vécue de façon douloureuse par beaucoup de familles, ce qui a conduit à l'adoption de la loi du 8 janvier $1993^{5}$ qui a créé un article 79-1 inséré dans le livre Ir du Code civil traitant des personnes, et plus précisément dans le Livre II consacré à l'état civil. 
Au terme de cet article 79-1 du Code civil:

"Lorsqu'un enfant est décédé avant que sa naissance ait été déclarée à l'état civil, l'officier de l'état civil établit un acte de naissance et un acte de décès sur production d'un certificat médical indiquant que l'enfant est né vivant et viable et précisant les jours et heures de sa naissance et de son décès. À défaut du certificat médical prévu à l'alinéa précédent, l'officier de l'état civil établit un acte d'enfant sans vie. Cet acte est inscrit à sa date sur les registres de décès et il énonce les jour, heure et lieu de l'accouchement, les prénoms et noms, dates et lieux de naissance, professions et domiciles des père et mère et, s'il y a lieu, ceux du déclarant. Lacte dressé ne préjuge pas de savoir si l'enfant a vécu ou non; tout intéressé pourra saisir le tribunal de grande instance à l'effet de statuer sur la question. »

Le premier alinéa de cet article permet clairement de donner un état civil complet aux enfants nés vivants et viables mais décédés avant leur déclaration de naissance. Un acte de naissance et un acte de décès sont alors dressés, même si l'enfant n'a vécu que très peu de temps. Ainsi, sa filiation peut être mentionnée et établie: ses noms et prénoms figurent dans l'acte, la législation funéraire peut s'appliquer (délivrance d'un permis d'inhumer, obsèques obligatoires, etc.) sous réserve que la naissance soit déclarée dans les trois jours de l'accouchement. À défaut du certificat médical constatant et la vie et la viabilité (art. 79-1, al. 2 du Code civil), seul peut être établi un acte d'enfant sans vie.

Il est clair qu'une fois encore toute la question tourne autour de la notion de viabilité. Jusqu'en 2008, les médecins, pour établir le certificat médical visé à l'alinéa 1, avaient recours à la notion telle que définie par l'Organisation Mondiale de la Santé (OMS) en 1977, à savoir vingt-deux semaines d'aménorrhée ou un poids de 500 grammes. Les juges, lorsqu'ils étaient saisis, respectaient eux aussi strictement ces critères, par ailleurs repris dans deux circulaires ministérielles des 3 mars 1993 et 30 novembre 2001 qui, selon les magistrats devaient servir à interpréter l'article 79-1 du Code civil. Pour les juges, la référence aux critères de l'OMS évitait que l'établissement d'un acte d'enfant sans vie ne dépende de l'opinion personnelle et donc subjective de l'officier d'état civil saisi ou de l'appréciation du médecin. Autrement dit, les enfants en deçà du seuil de viabilité défini par l'OMS ne pouvaient faire l'objet d'une déclaration d'enfant sans vie, tous les autres décédés au-delà de ce seuil pouvaient bénéficier de cet acte, s'ils n'étaient pas nés vivants et viables, un certificat médical devant en justifier.

En 2008, la situation a radicalement été modifiée suite à trois décisions rendues le même jour par la Cour de cassation sur la base de pourvois faisant suite à trois décisions de la Cour d’appel de Nîmes. Comme l'a fort bien résumé le Conseiller rapporteur chargé de l'affaire:

"Les pourvois qui nous sont soumis posent la question des conditions d'établissement d'un acte d'enfant sans vie, acte prévu à l'article 79-1 du code civil. Cet article prévoit-il un seuil en deçà duquel, un fotus ne pourrait pas faire l'objet d'un tel acte? Dans l'affirmative qui doit fixer ce seuil et comment le déterminer? Le respect dû à tout être humain dès le commencement de la vie s'applique-t-il à l'embryon et au foetus et dans l'affirmative, ce devoir de respect implique-t-il qu'un acte ou qu'un enregistrement ait lieu à l'état civil? »

La réponse de la Cour de cassation est dénuée de toute ambiguïté. Pour elle, « l'article 79-1, alinéa 2, du code civil ne subordonne l'établissement d'un acte d'enfant sans vie ni au poids du foetus, ni à la durée de la grossesse. » 
Dès lors, la viabilité n'est plus une condition nécessaire à l'établissement d'un acte d'enfant sans vie. Mais ce texte de l'article 79-1 s'applique-t-il dans tous les cas, notamment en cas d'interruption volontaire de grossesse? La question qui se profile derrière est de savoir s'il est légitime et logique d'inscrire sur un acte d'enfant sans vie un enfant dont la mère n'a pas voulu. Pour répondre à cette question, il convient de se reporter au texte du décret n 2008-800 du 20 août 2008 relatif à l'application de l'alinéa 2 de l'article 79-1 du Code civil qui dispose que:

« Lacte d'enfant sans vie est dressé par l'officier d'état civil sur production d'un certificat médical mentionnant les heures, jour et lieu de l'accouchement. »

Un arrêté, également du 20 août 2008, apporte des précisions sur la personne qui peut établir un tel acte. Cet arrêté renvoie en outre à un modèle de certificat médical qui comporte des indications sur les situations ouvrant droit à un tel certificat : sont clairement exclues deux hypothèses, celle des interruptions spontanées précoces de grossesse (fausse couche spontanée intervenant avant 22 semaines d'aménorrhée) et celle des interruptions volontaires de grossesse.

Maintenant que nous avons examiné la notion d'enfant sans vie, qui s'est affinée au cours du temps et qu'un acte lui est consacré, il reste à examiner quelle est la portée de cet acte. L'acte d'enfant sans vie est un simple acte enregistré par l'officier d'état civil, qui se borne à constater l'existence de l'enfant, mais qui ne lui confère aucun droit. Il s'agit donc d'un pur acte symbolique d'individualisation de l'enfant qui n'a aucune personnalité juridique. Cette individualisation se fait notamment par la possibilité offerte aux parents de donner des prénoms à l'enfant et de le faire figurer comme enfant sans vie sur le livret de famille. Faute de personnalité juridique, il n'est pas établi de filiation à l'égard d'un enfant sans vie et son décès ne peut donner lieu à ouverture d'une succession. Si l'acte d'enfant sans vie n'est pas générateur de droits vis-à-vis de l'enfant, il n'en va pas de même pour les parents puisque ceux-ci peuvent bénéficier, du fait de cet acte, d'un congé de maternité ou de paternité et obtenir le cas échéant des indemnités journalières.

En conclusion de tout ceci, il résulte clairement que l'enfant mort-né, ou enfant sans vie, est bien un reste, presque au sens mathématique, c'est-à-dire ce qui est en excès d'un dividende ou d'une opération. Il est un reste car il n'est « logeable » ni dans la catégorie des personnes, faute de pouvoir accéder à la personnalité juridique, ni dans celle des choses dans la mesure où il bénéficie en sa qualité « d'être humain » d'une protection et du respect au sens de l'article 16 du Code civil, ce que les lois bioéthiques viennent confirmer. Pourtant, il a bien existé et l'acte d'enfant sans vie vient en témoigner en lui conférant une certaine individualité à défaut de personnalité. Reste, encore, car, bien qu'individualisé, ce qui revient à lui reconnaître la qualité d'individu, mais non celle de personne, il ne peut prétendre être « fils de ». C'est un enfant sans filiation, un enfant qui ne s'inscrit pas dans une lignée et pourtant un enfant. C'est un reste car faute de personnalité juridique, il ne « reste » rien, juridiquement parlant, de sa courte existence, aussi brève et imparfaite fut-elle. Il ne reste qu'à titre de mémoire sur un livret d'état civil, c'est une « ombre de personne physique » pour reprendre l'expression de Gérard Farjat (2002 : 221), qui lui confère quand même le statut de « centre d'intérêt », comme peuvent l'être d'autres institutions qui ne sont pas nécessairement qualifiées juridiquement, telles que la famille, les groupes de société etc. dont l'existence ne doit rien à une volonté normative, notamment du législateur, (même si celui-ci peut réglementer leur fonctionnement), mais qui relèveraient d'un « ordre spontané », « résultats de l'action des hommes, mais 
non de leurs desseins » (ibid.: 223) D'autres juristes retiennent la qualification de «personne par destination », chose ou « produit » qui n'est pas encore une personne mais qui était appelée à le devenir. Mais que fait-on de ce reste, autrement dit quel traitement doit-on réserver au corps des enfants sans vie?

\section{Le traitement des restes de l'enfant sans vie}

Le Comité Consultatif National d'Éthique a rendu le 22 septembre 2005 un avis « relatif à la conservation des corps des foetus et des enfants mort-nés » (avis n $\left.{ }^{\circ} 89\right)^{6}$.

Pour le CCNE le fœutus est devenu, du fait des progrès médicaux « un être reconnu avant sa naissance », laquelle serait « un aboutissement plus que le début d'une existence ». Même si le droit établit le moment de la naissance comme une frontière venant délimiter ce qui relève de la personne et ce qui n'en relève pas, il n'y a pas lieu, pour le CCNE d'adopter une attitude binaire « de respect absolu dans un cas et de respect relatif dans l'autre, d'autant que cette frontière peut répondre à des âges chronologiques différents: une naissance prématurée suffit à transformer soudain un foetus en nouveau né ». Il résulte de ces considérations que les soignants « doivent prendre en compte le corps de ce fœetus ou de cet enfant mort-né ». Il recommande notamment de s'abstenir de toute conservation des corps des enfants nés sans vie à des fins de collections et en toute hypothèse au-delà du seul besoin d'effectuer des autopsies destinées à rechercher les causes de la mort dans le but d'éviter une nouvelle grossesse pathologique. En effet, il convient de respecter le souhait des familles qui peuvent envisager de pratiquer des rites funéraires. Toutefois, s'il n'est plus possible de conserver des collections, les parties du corps « non identifiantes » telles que viscères, cerveau, etc., peuvent être conservées « en fonction des nécessités scientifiques et des protocoles de recherche » mais toujours « après autorisation parentale ».

Le CCNE établit, à propos des rites funéraires, une distinction entre les enfants nés vivants et viables et les enfants sans vie. Pour les premiers, nous sommes en présence d'un enfant mineur dont le sort relève de la seule volonté des parents qui ont l'obligation de prendre en charge l'inhumation ou la crémation de l'enfant, à leurs frais. Pour les autres, la crémation ou l'inhumation n'est pas obligatoire. Si les parents décident d'y procéder, ils prennent en charge les frais afférents et peuvent être aidés par la commune en cas de difficulté. Toutefois, en cas d'impécuniosité des parents, la commune doit prendre en charge les obsèques (cf. circulaire n 2001/576 du 30 novembre 2001, BO 2001 Nº 20). Dans tous les cas, il conviendra d'en passer par un organisme de pompes funèbres si les parents décident d'inhumer ou d'incinérer l'enfant. L'établissement doit informer les parents des possibilités qui leur sont offertes. Ceux-ci disposent alors d'un délai de dix jours pour se déterminer. Passé ce délai, si les parents n'ont pas fait connaître leur volonté ou s'ils n'ont pas souhaité réclamer le corps, celui-ci fait l'objet d'une incinération collective dans un crématorium autorisé conformément à l'article L. 2223-40 du Code général des collectivités territoriales et dont le gestionnaire est titulaire de l'habilitation
Tableau des étapes embryonnaires (Fondation Carnegie)

Ces clichés sont un système utilisé par des embryologistes pour décrire la maturité apparente d'embryons.

๑ http://embryo.soad.umich.edu/ carnStages/carnStages.html 
prévue à l'article L. 2223-4l de ce code. La crémation est à la charge de l'établissement de santé. Il est clair, pour le CCNE que « le désir des parents concernant le devenir du corps du fotus ou du nouveau né doit toujours être respecté quel qu'il soit ».

En cas d'autopsie, le corps doit être remis à la famille s'il est réclamé, mais après restauration. Il ressort clairement de ce qui précède que la tendance est, de plus en plus, à considérer ou permettre aux parents de considérer, que le corps de leur enfant né sans vie, même s'il n'a pas atteint un stade de développement important est un cadavre qui doit être traité comme tel, selon les procédures et les rites habituels. De surcroît, la pratique hospitalière introduit une sorte d'obligation de proposer aux parents de voir le corps et d'organiser des obsèques (Memmi 2011 : 71) faisant sortir la vue de ces corps du seul champ professionnel, puisque jusqu'à présent les cadavres de ces enfants étaient soustraits à la vue des proches, seule l'équipe soignante étant confrontée à ces dépouilles. Toutefois, cette possibilité n'est pas offerte pour les corps des enfants issus d'une IVG ou d'une interruption spontanée précoce de grossesse. Que pouvons-nous tenter de dégager de tout ceci?

\section{Le « travail de deuil », une norme pour tenter d'apprivoiser la mort?}

Il est frappant de constater le traitement paradoxal réservé à ces cadavres d'enfants, ces « restes » puisque c'est le terme que nous avons retenu, selon qu'il s'agisse des restes d'un avortement ou d'une fausse couche, ou de ceux d'un « enfant sans vie » au sens où nous l'avons défini.

En effet, d'un côté, la pratique médicale vise à désincarner l'enfant in utero afin que la mère ne puisse s'y attacher, certainement avec l'idée que plus le fotus sera désincarné moins la culpabilité possible sera importante. De l'autre, il s'agit au contraire de " présentifier » l'enfant, soit en présentant effectivement le corps de celui-ci, soit, si cette présentation n'est pas possible, de présenter des signes de l'enfant, de jouer sur la représentation ou la métonymie en montrant des photographies du fotus prises lors d'examens périnataux ou des objets destinés à l'enfant à naître.

Il est intéressant également de souligner qu'il s'agit bien là d'une pratique médicale, d'une pratique de l'institution qui consiste à mettre en scène, à rendre visibles (ou au contraire à rendre invisibles) ces enfants morts, comme s'il s'agissait à cette occasion de leur conférer une certaine réalité, une substance que sans cela, faute d'être nés, ils n'auraient pas eue. Ceci renoue avec une pratique ancienne (Morel 2001 : 17-38), qui était antérieurement le fait des familles et des proches et qui aurait perduré en France jusque vers 1950 (ibid. note 35), consistant à mettre en scène les enfants morts. La différence est qu'il s'agit dorénavant de la mise en scène des foetus, à d'autres fins que celles d'illustration médicale. À ce propos, il est frappant de noter le parallèle entre le développement de l'imagerie médicale, qui permet d'obtenir des images d'enfants vivants in utero et le développement de cette pratique de mise en œuvre d'une image de l'enfant mort-né (Memmi 2011 : 65), pratique qui, au demeurant semble réservée à ces enfants, sans concerner les adultes (Memmi 2011 : 66-sqq). Faut-il s'en étonner? Il semble que 


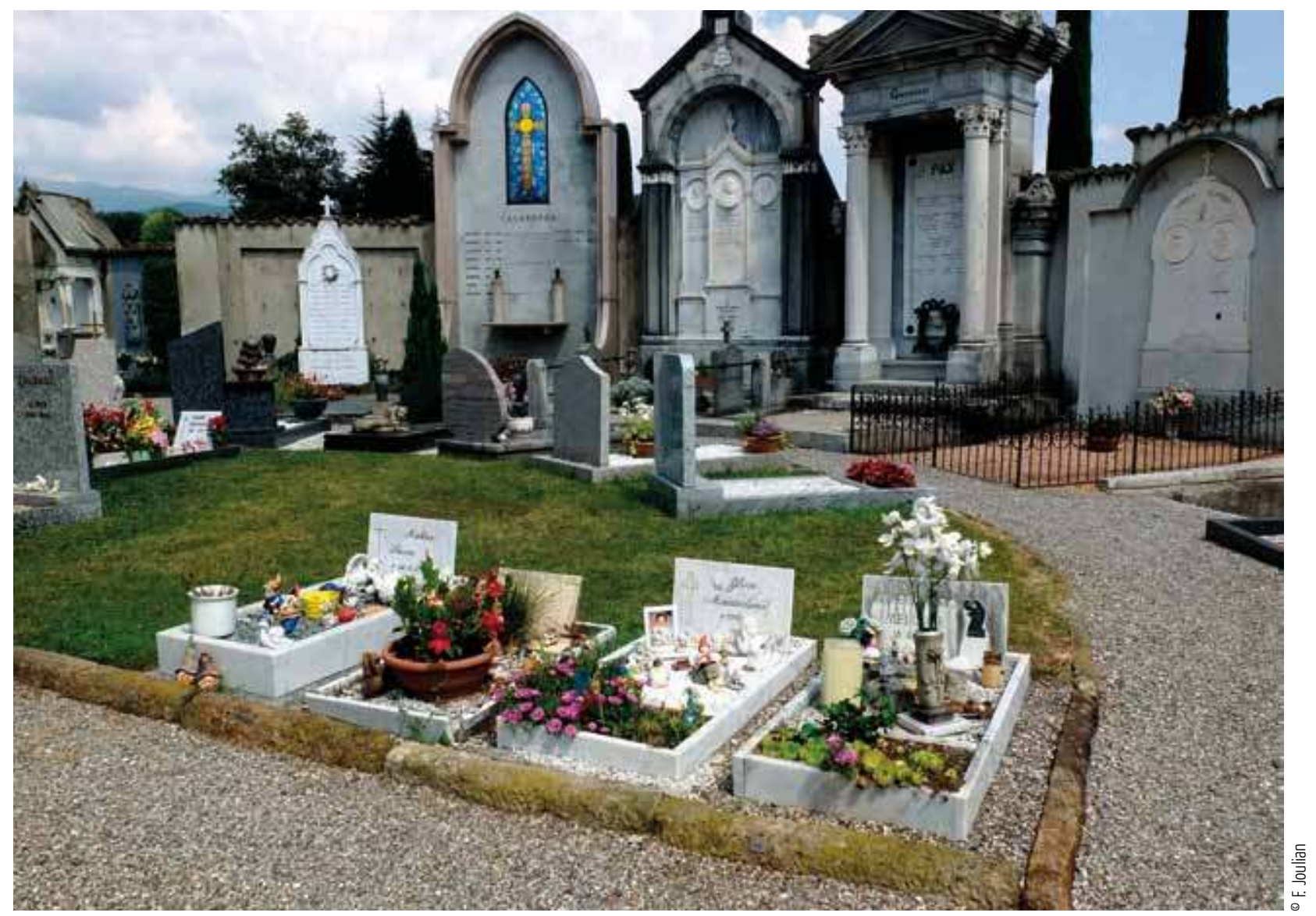

non. En effet, le décès d'un enfant vient interrompre quelque chose de la lignée familiale. Il y a soudain, dans la série dans laquelle s'inscrivent parents, enfants et petits-enfants, quelque chose qui vient briser la chaîne, remettre en cause la logique qui voudrait que les plus âgés meurent les premiers. La mort d'un enfant c'est une transmission qui ne se fait pas, un avenir qui s'efface.

Par ailleurs, l'enfant mort-né nous place directement, immédiatement, face au fait que la question de la mort est au centre de la question de la procréation, puisque donner naissance c'est faire naître à la mort dans la mesure où tout enfant qui naît est un défunt en devenir, à plus ou moins longue échéance. Cette question vient nous heurter, précisément parce qu'il y a, avec le décès d'un enfant, quelque chose de la temporalité qui est perturbé, un « trop tôt » qui vient se mettre en place et qui laisse les vivants désemparés. Mais avec l'enfant mort-né, ce n'est plus un « trop tôt » qui survient, c'est un véritable télescopage temporel, puisque l'enfant est mort avant même d'être né. Le « trop tôt » est antérieur à toute naissance et donc à toute vie perceptible extra utero.

La question qui subsiste toutefois est celle de savoir en quoi cette pratique hospitalière qui incite à voir les enfants mort-nés pourrait être [socialement? psychologiquement? éthiquement? utile? Ce qui légitime et fonde cette pratique est un a priori selon lequel les parents pourraient accéder plus aisément au «travail du deuil ${ }^{7}$ » lorsqu'ils voient

\section{Tombes d'enfants mort-nés}

Sur cette photo prise dans un cimetière du Tessin, les enfants mortnés sont désignés par une seule date de naissance et de mort.

Ils sont inhumés dans un carré spécifique de l'allée centrale. 
l'enfant, ce qui permettrait de lui conférer réalité et donc une identité et donc de faire en sorte que malgré cette mort prématurée, les parents se sentent effectivement parents. Un tel a priori semble contestable car il met l'accent sur le biologique, là où être parent ne relève pas essentiellement du biologique. Cette concrétisation, voire cette biologisation de la fonction parentale peut être mise sérieusement en question. Il n'en demeure pas moins que de se trouver confronté à une telle expérience n'est pas chose facile pour les parents, et notamment les mères. Cette question de l'identité est à mettre en parallèle avec la norme juridique consistant à conférer à l'enfant mort-né une place dans le livret de famille sans toutefois lui conférer une véritable identité.

Cette place particulière est une inscription qui rappelle aux parents, s'ils le souhaitent, qu'un enfant est venu pendant un temps très court s'intercaler au moins potentiellement dans la chaîne générationnelle, sans toutefois y prendre place. Mais cette inscription est-elle nécessaire pour que les parents se souviennent de ce fait? Ne ravive-t-elle pas sans fin le souvenir, une fois de plus, en présentifiant par l'inscription, ce qui devrait relever du travail de mémoire, c'est-à-dire d'un travail qui sélectionne subjectivement les faits pour n'en retenir que certains, ou certains aspects, tout aussi subjectivement? La question mérite semble-t-il d'être posée. Elle le mérite d'autant plus que si l'on se réfère au travail de Freud sur le deuil, dont se réclament certains tenants ou promoteurs des pratiques décrites, celui-ci est loin du volontarisme, loin d'un quelconque travail à faire. Pour Freud en effet, le deuil n'est pas un état pathologique qu'il conviendrait de traiter $^{8}$, il est au contraire un état «normal » et si travail il y a, c'est celui du temps et Freud ajoute même que toute intervention, toute perturbation de ce travail du temps est à éviter: "Nous comptons bien qu'il [le deuil] sera surmonté après un certain temps, et nous considérons qu'il serait inopportun et même nuisible de le perturber. » (Freud [1915] 1968 : 1146).

Photographies, vêtements n'ayant pas pu servir, mèches de cheveux, voire inscription sur un livret de famille ne sont-ils pas autant de fétiches qui s'opposent au deuil, car s'il est un travail que le deuil peut permettre d'accomplir, c'est celui de consentir à la perte (Allouche 1995 : 140-141)? Il semble bien au contraire que la seule chose qui puisse apaiser serait, non pas cette présentification, parfois permanente, de l'enfant mort-né au travers de ce qu'on peut appeler des fétiches, mais bien la mise en place d'un rituel, celui de l'inhumation ou de la crémation. Le rituel, en effet, est une des choses qui face au non-sens radical de la mort, peut venir apaiser la quête incessante du sens qui anime tout être parlant. Enfin, nous pouvons affirmer que les théories prescriptives sur le deuil, à l'œuvre tant dans les milieux hospitaliers que dans la pratique administrative et jusque dans les textes législatifs, concourent à tenter de normativer, voire de normaliser la mort et le deuil. Il s'agirait pour «faire son deuil » d'en passer par des étapes prédéfinies, des modèles ou des processus, comme si l'être humain pouvait, dans sa singularité, se résumer à des processus quasi mécaniques. Il n’y a pas de « bon deuil ». 


\section{$\&$}

La question de l'enfant mort-né nous place donc au cœur de l'humain et de façon peut être plus violente qu'à l'occasion de la mort d'un enfant qui a pu vivre quelque temps. Elle nous confronte en effet non seulement à la question de la filiation et de la transmission, mais également à celle de la mort, dans un raccourci temporel saisissant qui fait passer, en un instant du futur, ou du conditionnel au plus-que-parfait. 


\section{NOTES}

Photo d'ouverture : «Ombre d'être humain » (c) Dessin original de Nicolas Weydert 2013.

1. Avis n 1 du CCNE, 22 mai 1984.

2. Art. 318,725 et 906 du Code civil.

3. Déclaration du Roy, Concernant la forme de tenir les registres de Batêmes, Mariages, Sepultures, Vestures, Noviciats \& Professions; Et des Extraits qui en doivent être delivrez. Donnée à Versailles le 9 avril 1736. Registrée en Parlement, consultable à l'adresse: http:// fr.wikisource.org/wiki/D \% C3 \% A9claration_du_ Roy_du_9_avril_1736, consulté le 6 décembre 2013. Cette déclaration précise notamment les règles de tenue des registres précédant la création de l'état-civil.

4. Décret du 4 juillet 1806 contenant le mode de rédaction de l'acte par lequel l'officier de l'état civil constate qu'il lui a été présenté un enfant sans vie, consultable sur le site www.legifrance.gouv.

5. Loi n 93-22 du 8 janvier 1993 modifiant le code civil, relative à l'état civil, à la famille et aux droits de l'enfant et instituant le juge aux affaires familiales, (article 6), JORF 9 janvier 1993, p. 495.
6. http://www.genethique.org/carrefour_infos/textes_ officiels/titres_textes/textes/ccne_avis089_conservation_foetus.pdf, (consultable en décembre 2011; cette page n'est plus consultable aujourd'hui 6 décembre 2013).

7. Notons que ce terme est issu du texte de S. Freud «Deuil et mélancolie » (in Metapsychologie) paru en 1915, essentiellement consacré à la pathologie de la mélancolie, qui, en psychopathologie, est à distinguer de la dépression. Cependant, le terme de travail de deuil (Traurerarbeit) n'apparaît qu'une seule fois dans ce texte et nulle part ailleurs dans les écrits de Freud. La notion de «travail de deuil » est donc une construction de la psychologie (et non de la psychanalyse) contemporaine.

8. Même s'il existe des deuils pathologiques, mais ceuxci sont à distinguer de ce qu'on appelle le deuil au sens général.

\section{POUR CITER CET ARTICLE}

Gasnier, J. - P. 2013 L'Enfant mort-né, du futur au plus-que-parfait, in H. Guy, A. Jeanjean \& A. Richier, Le Cadavre en procès, TechniquesÉCulture $60: 30-43$.

\section{RÉFÉRENCES}

Allouche, J. 1995 Érotique du deuil au temps de la mort sèche. Paris: Epel: 140-14l.

Farjet, G. 2002 Entre les personnes et les choses, les centres d'intérêts. Prolégomènes pour une recherche, Revue Trimestrielle de Droit Civil (RTD Civ.) 2002 : 221.

- 2002 Entre les personnes et les choses, les centres d'intérêts. Prolégomènes pour une recherche, Revue Trimestrielle de Droit Civil (RTD Civ.) 2002 : 223.

Freud, S. [1915] 1968 Deuil et mélancolie. In Metapsychologie. Paris: Gallimard.

Memmi, D. 2011 La Seconde vie des bébés morts. Paris: Éditions de l'EHESS (Cas de figure) : 71.

Morel, M.-F. 2001 Images du petit enfant mort dans l'histoire. In Études sur la mort: le deuil d'enfants de la conception à la naissance, L'Esprit du temps (119) : 17-38 (cité in Memmi D. 2011 La Seconde vie des bébés morts. Paris: Éditions de l'EHESS (Cas de figure) : 60 - note 32.

Murat, Ph. 1995 Décès périnatal et individualisation juridique de l'être humain, Revue de droit sanitaire et social (RDSS): 451. 


\section{RÉSUMÉ}

L'Enfant mort-né, du futur au plus-que-parfait. Le propre de l'activité juridique est de qualifier les choses, les personnes, les faits, les situations. Qualifier un enfant mort-né n'est pas simple et dépend de la réponse à certaines questions, notamment: qu'est ce qu'un être humain? Peut-on appliquer cette qualification à un embryon? qu'est-ce qu'être viable? Les réponses à ces questions sont plus ou moins influencées par des prises de positions religieuses, philosophiques, morales, épistémologiques ou anthropologiques et de ces réponses découlent diverses conséquences juridiques, dont celle, non dénuée d'intérêt, de savoir si l'enfant a une existence légale ou non.

Face aux évolutions de la société le droit a façonné une qualification nouvelle, celle d'enfant sans vie de laquelle découlent certaines conséquences juridiques, sans pour autant que cet enfant sans vie ait une quelconque existence légale. Qu'est-ce donc qu'un enfant sans vie au regard des qualification juridiques classiques? « Ombre d'être humain », ni chose, ni personne, quel traitement lui est réservé? Que penser de ces nouveaux traitements qui tendent à se normaliser dans la pratique hospitalière et qui ont pour but d'aider à « faire le deuil »?

C'est à ces questions, notamment, que cet article tente de répondre, en soulignant que la question des enfants sans vie nous confronte non seulement à la question de la filiation et de la transmission, mais également à celle de la mort, dans un raccourci temporel saisissant qui fait passer en un instant du futur ou du conditionnel au plus-que-parfait.

\section{ABSTRACT}

Child born without life, of future in the past perfect. The own of the legal activity is to qualify things, people, facts, situations. To qualify a stillborn child is not simple and depends of the answer to certain questions, in particular: what 's a human? Can we apply this qualification to an embryo? What to be "viable »? The answers to these questions are more or less influenced by religious, philosophic, moral, epistemological or anthropological stands and of these answers ensue diverse legal consequences, among which that, not divested of interest, to know if the child has a legal existence or not.

In front of evolutions of the society the law shaped a new qualification, that from the dead child of which ensue certain legal consequences, however, it does not mean that this dead child has any legal existence. What is it thus that a dead child with regard to qualification legal classic? Human being's « shadow », neither thing, nor anybody, what treatment processing is reserved for them? That to think of these new treatments which tend to standardize in the hospital practice and which aim at helping « to make the mourning »?

It is these questions, in particular, that this article tries to answer, by underlining that the question of the dead children confronts us not only with the question of the filiation and the transmission, but also with that of the death, in a striking temporal shortcut which makes pass in an instant of future or of the conditional in most that completed.

\section{MOTS CLÉS}

mort-né, déchet, embryon, cadavre, deuil, viabilité

\section{KEYWORDS}

stillborn, waste, embryo, corpse, mourning, viability 